\title{
Analytical Performance and Characterization of a Quartz Crystal Microbalance for the Detection of $\mathrm{Cu}(\mathrm{II})$ lons in Water
}

\author{
Chi-Yen Shen ${ }^{1}$, Roan Yeh ${ }^{1}$, Mei-Hui Chung ${ }^{2}$, Rey-Chue Hwang ${ }^{1,}$, \\ ${ }^{1}$ Department of Electrical Engineering, I-Shou University, Kaohsiung, Taiwan \\ ${ }^{2}$ Office of Library and Information Services, I-Shou University, Kaohsiung, Taiwan
}

\section{Email address:}

cyshen@isu.edu.tw (Chi-Yen Shen), popokago0306@gmail.com (Roan Yeh), mhchung@isu.edu.tw (Mei-Hui Chung), rchwang@isu.edu.tw (Rey-Chue Hwang)

${ }^{*}$ Corresponding author

\section{To cite this article:}

Chi-Yen Shen, Roan Yeh, Mei-Hui Chung, Rey-Chue Hwang. Analytical Performance and Characterization of a Quartz Crystal Microbalance for the Detection of $\mathrm{Cu}$ (II) Ions in Water. Journal of Electrical and Electronic Engineering. Vol. 4, No. 5, 2016, pp. 103-108. doi: $10.11648 /$ j.jeee.20160405.13

Received: September 22, 2016; Accepted: October 14, 2016; Published: October 20, 2016

\begin{abstract}
A novel quartz crystal microbalance (QCM) sensor based on combining phosphate-modified dendrimer and ionophore has been developed for the determination of $\mathrm{Cu}(\mathrm{II})$ ions. The performance of the developed QCM sensor was evaluated based on frequency data and experimental results evidently indicated that the prepared sensor could be sensitive for the determination of $\mathrm{Cu}(\mathrm{II})$ ions in water. The obtained QCM sensor presents good selectivity monitoring of $\mathrm{Cu}(\mathrm{II})$ ions, short response time $(40 \mathrm{~s})$, and wide linear range $(0.01-100 \mu \mathrm{M})$.
\end{abstract}

Keywords: Quartz Crystal Microbalance, Metal Ion, Sensitivity, Selectivity

\section{Introduction}

The quartz crystal microbalance (QCM) is a well-established tool in mass-sensitive detection. These devices typically consist of an AT-cut quartz crystal with electrodes patterned on opposite sides. An electrical field, applied to the QCM, produces mechanical stresses that induce acoustic wave to travel in a direction perpendicular to the surfaces of the crystal. Therefore, QCM that can be electrically excited into a shear mode of vibration could be used to probe interfacial processes on quartz surface.

The QCM can consequently detect mass changes due to these molecular interactions on the surface of the QCM. The potential of QCMs for chemical sensor was an application of the Sauerbrey [1], who first described the relationship between the frequency shift and mass change on the crystal surface in air. The Sauerbrey equation is defined as:

$$
\Delta f=-\frac{2 f_{o}^{2} \Delta m}{A\left(\rho_{q} \mu_{q}\right)^{1 / 2}}
$$

where $\Delta f$ is the measured frequency shift, $f_{0}$ is the fundamental resonant frequency, $\Delta m$ is the mass change due to surface deposition, $A$ is the piezoelectrically active crystal area defined by the two gold electrodes, $\rho_{q}$ is the density of quartz crystal and $\mu_{q}$ is the shear modulus. Eq. 1 is developed for oscillation in air and only applies to rigid masses attached to the crystal. It is applicable to only gas phase mass deposition. If the solution passes over the QCM crystal surface, the frequency response of the QCM is dependent on bulk liquid properties such as conductivity, viscosity, density and dielectric constant $[2,3]$. The Kanazawa and Gordon's equation describes the oscillating frequency in liquid phase as follows:

$$
\Delta f=-f_{o}^{3 / 2}\left(\frac{\eta_{l} \rho_{l}}{\pi \rho_{q} \mu_{q}}\right)^{1 / 2}
$$

where $\rho_{l}$ is the density of the liquid and $\eta_{l}$ is the viscosity of the liquid. The QCM device is convenient to use and could rapidly detect real-time responses of chemical interactions on the surface of device $[4,5]$. Therefore, the low cost and easy operated QCM device has been applied in various biotechnology and chemical fields, such as clinical diagnosis [6-8] and environmental monitoring [9]. In this study, a 
simple QCM-based liquid sensor was developed to monitor metal ions without complex procedures. The efficiency, sensitivity and selectivity of the sensor were also evaluated to further enhance its practicability in drinking water.

Heavy metals are naturally occurring elements that have a high atomic weight and a density at least 5 times greater than that of water [10]. Several factors including the dose, route of exposure, and chemical species influence the degree of toxicity. The overall toxicity also depends on the age, gender, genetics, and nutritional status of exposed individuals. Among heavy metals, arsenic, cadmium, chromium, lead, and mercury reduce significant health risks due to their high degree of toxicity. These metallic elements are considered systemic toxicants that are known to induce multiple organ damage, even at lower levels of exposure [10].

The multiple applications in industry, agriculture, medicine, and technology widely use chemical products to result in a wide distribution of chemicals in the environment. These results raise concerns over potential effects of chemicals on human health and the environment [10-12]. Therefore, the detection of heavy metals in aqueous environments has attracted wide attention in recent years. Detection and quantification of heavy metals have been investigated by utilizing atomic absorption spectroscopy [8, 9], high performance liquid chromatography $[10,11]$, inductively coupled plasma-mass spectrometer $[12,13]$, and anodic stripping voltammetry [14]. However, such analytical methods have been found to have practical limitations with respect to cost, complexity and the requirement for a well-trained operator. Therefore, a variety of rapid, sensitive sensor has been developed for detecting metal ions in order to overcome these limitations. Many kinds of sensors have been studied for heavy metals determination, such as electrochemical sensors [20] and biosensors [21].

In this study, detection of $\mathrm{Cu}$ (II) ions in water has been investigated. Among heavy metal ions, copper has become a widely distributed pollutant in natural water as a result of the dumping of electronic trash and mining residues [21]. On the other hand, $\mathrm{Cu}$ (II) is the third most abundant transition-metal ion in the human body and is essential for the biochemical and physiological functions [22]. Its deficiency and excess lead to malfunction of the liver, heart diseases, neurological disorders, and deterioration of connective and bone tissues [10].

Dendrimers are branched polymers with well-defined sizes and geometries. Dendrimers with multiple surface functional groups are a new attractive class of macromolecules [23-25]. The ability of dendrimers to coordinate metal ions in their interior branches or in their exterior units can be exploited to develop sensors of high selectivity and sensitivity. The structural features of the multiple branches provide many active sites, which can bond with targets at many points. In addition, phosphate anions can exhibit high affinity for various types of metal ions and have been applied for ion sensing [26, 27]. o-Xylylenebis (N,N-diisobutyldithiocarbamate) is copper(II) ionophore I and forms chelates with copper of high stability as compared to other metals. Hence, taking these advantages into consideration, we designed and synthesized a new film of phosphate-modified dendrimer (A) incorporating PVC based on copper(II) ionophore I (B) as a sensing membrane. In this paper, a QCM sensor coated with this new membrane has been developed for the detection of $\mathrm{Cu}$ (II) metal ion in water. The detection responses of sensor on $\mathrm{Cu}$ (II) metal ion sensing performance were studied quantitatively.

\section{Experimental Methods}

\subsection{Reagents}

All reagents were of analytical reagent grade and were used without further treatment. PAMAM dendrimer (ethylenediamine core, G5), polyvinyl chloride (PVC), tetrahydrofuran (THF), and copper(II) ionophore I were purchased from Sigma-Aldrich, USA. Dimethylformamide (DMF) and phosphoric acid were obtained from Fluka and Kojima Chemical Co., Japan, respectively. All aqueous solutions were prepared with doubly deionized, distilled water.

\subsection{Preparation of Sensing Membrane}

$100 \mu \mathrm{L}$ of $5 \mathrm{wt} \%$ PAMAM dendrimer (ethylenediamine core, G5), whose chemical structure is shown in Figure 1, was dispersed in $900 \mu \mathrm{L}$ of $30 \%$ phosphorous acid solution in an ultrasonic bath for $7.5 \mathrm{~h}$ to produce phosphate-modified dendrimer (A) and then rinsed by deionized water. $1 \mathrm{mg}$ copper(II) ionophore I was dissolved in $50 \mu \mathrm{L}$ of THF and was agitated in an ultrasonic bath for $30 \mathrm{~min} .0 .1 \mathrm{~g}$ PVC was dissolved in $4.8 \mathrm{~mL}$ of DMF and was agitated in an ultrasonic bath for $20 \mathrm{~min}$. Then, the mixture of $1 \mathrm{~mL}$ PVC solution and $50 \mu \mathrm{L}$ ionophore solution was agitated in an ultrasonic bath for $1 \mathrm{~h}$ to produce PVC based on copper(II) ionophore I (B). The ion selective membrane solution was obtained mixing materials $\mathrm{A}$ and $\mathrm{B}$ at a specific ratio in an ultrasonic bath for $30 \mathrm{~min}$.

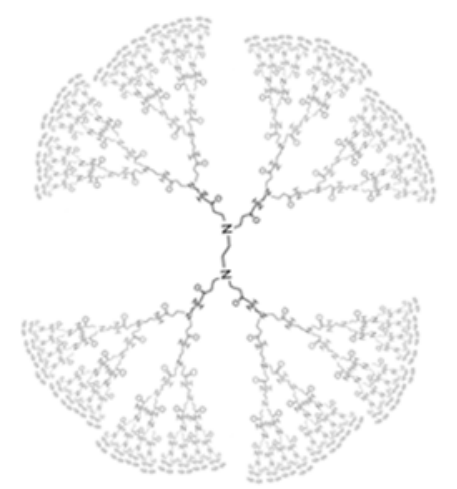

Figure 1. Chemical structure of ethylenediamine core G5 PAMAM dendrimer.

\subsection{Preparation of the QCM Sensor}

The QCM device (Taitien Co., Ltd, Taiwan) with an operation frequency of $10 \mathrm{MHz}$ was used in this work. QCM samples were first cleaned with piranha solution for $30 \mathrm{~s}$. A 
drop of $5 \mu \mathrm{L}$ sensing membrane solution was dropped on the electrode surface of each QCM sample at room temperature. Then the QCM samples were dried in a vacuum oven at $80^{\circ} \mathrm{C}$. Finally, the samples were rinsed again with deionised water and dried with $\mathrm{N}_{2}$ gas.

The fabrication procedure of a QCM sensor based on ion selective membrane is illustrated in Figure 2 and a real image of a sensing membrane coated QCM sensor is shown in Figure 3.

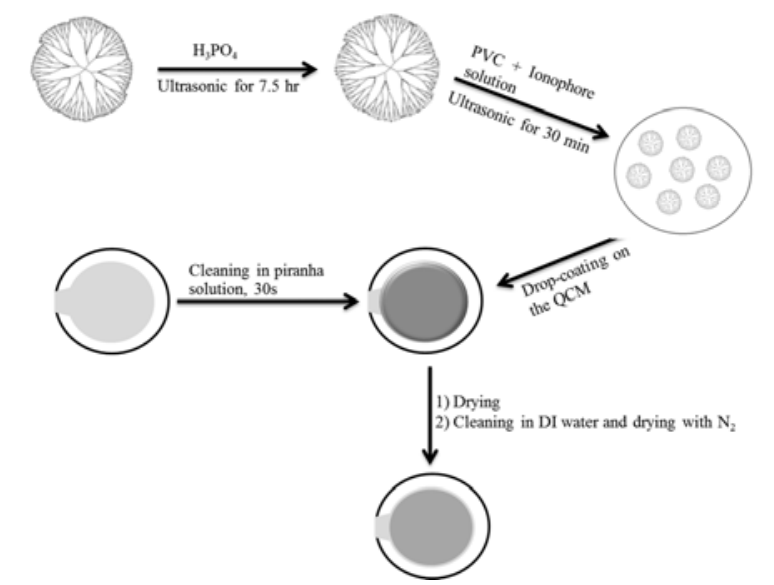

Figure 2. Schematic of the fabrication procedure of a QCM sensor.

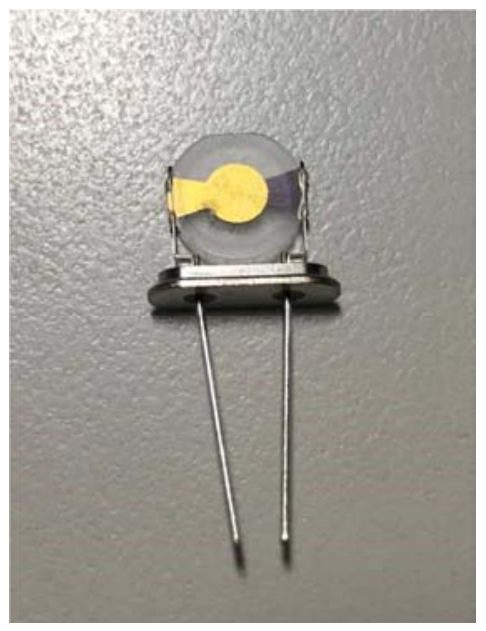

Figure 3. A photograph of a QCM sensor.

\subsection{Apparatus}

An apparatus shown in Figure 4 was used to monitor metal ions in this study. A counter (53131A, Agilent, USA) measured oscillation frequency of the QCM device. A computer through a GPIB interface controlled the counter, and the software for taking measurements was designed by LabVIEW 8.6 (National Instruments, USA). Figure 5 shows that a QCM sensor was mounted by concentric rubber seals into a flow cell to provide contact with only one side of the quartz crystal to the liquid. Solutions were injected into the flow cell by two syringe infusion pumps (KDS 200, KD Scientific Inc., USA), through $0.5 \mathrm{~mm}$ diameter silicon tube for liquid exchange, as shown in Figure 4. The cell was shielded with a temperature controller box that was maintained at $20^{\circ} \mathrm{C}$.

The QCM sensor was designed to make use of the Pierce oscillator circuit plotted in Figure 6 to generate the oscillation in this study. The design of a crystal-controlled oscillator used as a QCM sensor in liquid is a difficult task because of the wide dynamic values of the resonator resistance that they should support during their operations [28]. The QCM experiences a large reduction in its quality factor that caused by the liquid. The Pierce oscillator design provides a great stability in frequency and a low phase noise. Output spectrum of the QCM oscillator measured by the spectrum (Model 4395A, Agilent, USA) is shown in Figure 7 and indicates QCM generated stable oscillation occurred at $10 \mathrm{MHz}$.

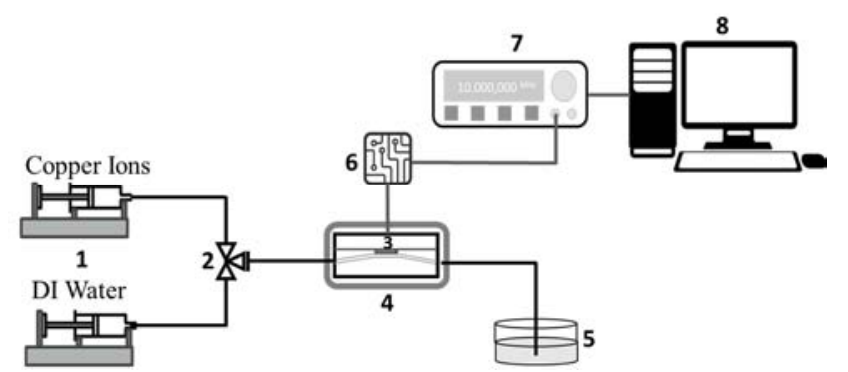

Figure 4. Diagram of the experimental system: 1: syringe pumps; 2: flow valve; 3: QCM; 4: temperature-controlled flow cell; 5: waste; 6: oscillator circuit; 7: frequency counter; 8: computer.

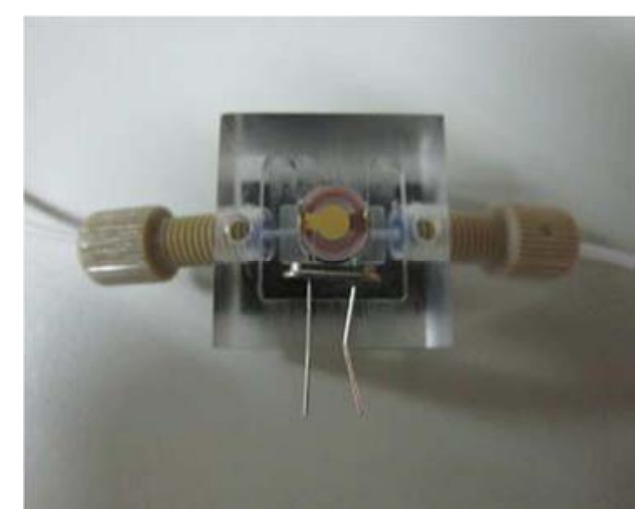

Figure 5. A QCM sensor was mounted by concentric rubber seals into a flow cell.

\section{U1}

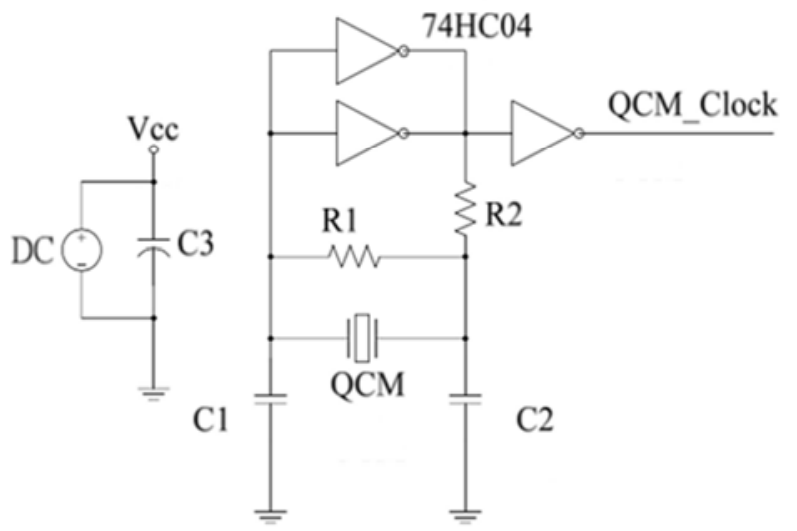

Figure 6. The circuit diagram of the Pierce oscillator [29]. 


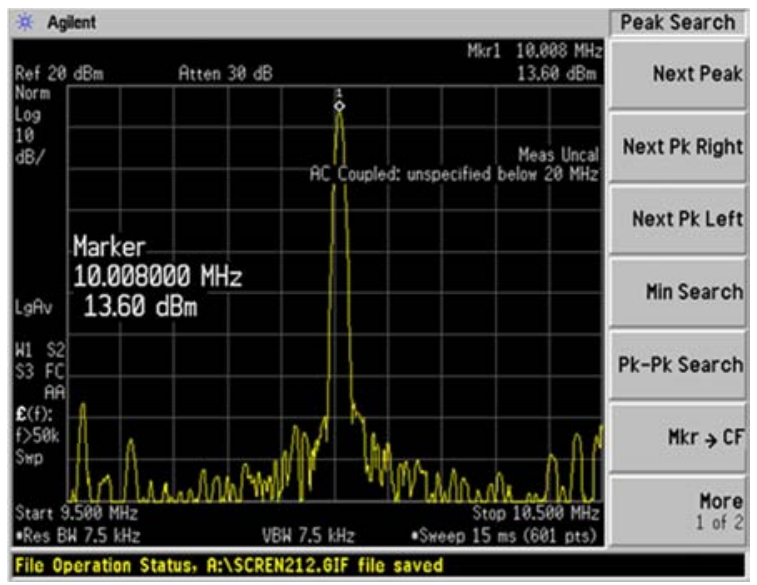

Figure 7. Output spectrum of the QCM oscillator in this work [29].

\subsection{Procedures for Metal Ion Detection}

Various concentrations of metal ion solutions were prepared by mixing in various ratios with the developed. During the detection, the developed and $300 \mu \mathrm{l}$ diluted metal ion solution were injected into the flow cell carefully with two syringe infusion pumps, respectively, at $0.01 \mathrm{ml} / \mathrm{min}$. After the frequency signal stabilized for several minutes, the QCM sensor generated a frequency-decrease after metal ions bounded with the function groups in the surface of sensing membrane. The frequency shifts in all experiments were calculated based on the average responses of the reactions with corresponding standard deviations of triplicate measurements.

\section{Results and Discussion}

\subsection{Effect of pH on Response of the QCM Sensor}

The effect of the $\mathrm{pH}$ of the solution on the response of the QCM sensor with a PVC based on copper(II) ionophore I (B)/ phosphate-modified dendrimer (A) volume ratio of 9 was studied by varying the $\mathrm{pH}$ in $1 \mu \mathrm{M} \mathrm{Cu}$ (II) ion. The results are shown in Figure 8. The frequency shift of the QCM sensor increased with $\mathrm{pH}$ from 5 to 5.7 , and decreased as the $\mathrm{pH}$ was increased further. A maximum frequency shift was observed in the $\mathrm{pH}$ 5.7. Therefore, the solution with $\mathrm{pH}$ 5.7 was used to study further the sensing properties.

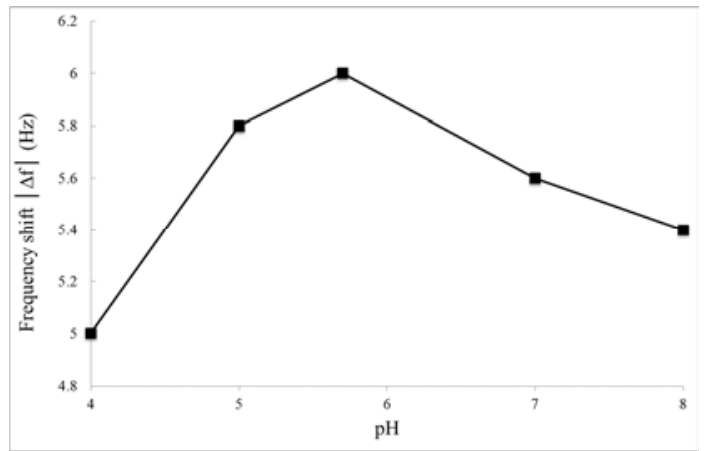

Figure 8. Effect of $p H$ on the frequency shift of the QCM sensor in $1 \mu M$ $\mathrm{Cu}(\mathrm{II})$ ion.

\subsection{Effect of Volume Ratio of PVC Based Material on Copper(II) Ionophore I (B) to Phosphate-Modified Dendrimer (A) on Sensitivity and Selectivity of Cu(II) Detection}

The performance of QCM sensors that were coated with sensing membrane with various volume ratios of B to A was studied and the results are shown in Table 1. Selective recognition of the target ions is an important characteristic of a QCM sensor. The selectivity factor is defined as the change in the ratio of the resonant frequency of the sensor in response to an interfering metal ion to that in response to a $\mathrm{Cu}$ (II) ion. The $\mathrm{Zn}$ (II) ion was selected as the competitor species because it has the same charge and a similar size. The thin film with a B/A volume ratio of 9 had higher selectivity than that with other ratios (Table 1). At a B/A volume ratio of 9, the prepared QCM sensor also exhibited high sensitivity for $\mathrm{Cu}(\mathrm{II})$ detection.

Figure 9 shows the selectivity to various metal ions of the sensing membrane with a $\mathrm{B} / \mathrm{A}$ volume ratio of 9 . The sensing membrane with a B/A volume ratio of 9 also exhibited high selectivity for $\mathrm{Cu}(\mathrm{II})$ in the presence of other metal ions. The high selectivity is believed to arise from the contribution of copper(II) ionophore I.

Table 1. Effect of volume ratio of B to A on performance of QCM sensor.

\begin{tabular}{llll}
\hline B/A & $4 / 6$ & $8 / 2$ & $9 / 1$ \\
Sensitivity $^{\mathrm{a}}(-\Delta \mathrm{f}, \mathrm{Hz})$ & 4.2 & 7.2 & 17 \\
Selectivity $^{\mathrm{b}}$ & 0.8 & 0.6 & 0.5 \\
\hline
\end{tabular}

${ }^{\text {a }}$ Sensitivity was defined as the frequency shift to $10 \mu \mathrm{M} \mathrm{Cu}$ (II) ion.

${ }^{\mathrm{b}}$ Selectivity was defined as the change in the ratio of the resonant frequency of the sensor in response to $\mathrm{Zn}$ (II) ion to that in response to a $\mathrm{Cu}$ (II) ion at $10 \mu \mathrm{M}$.

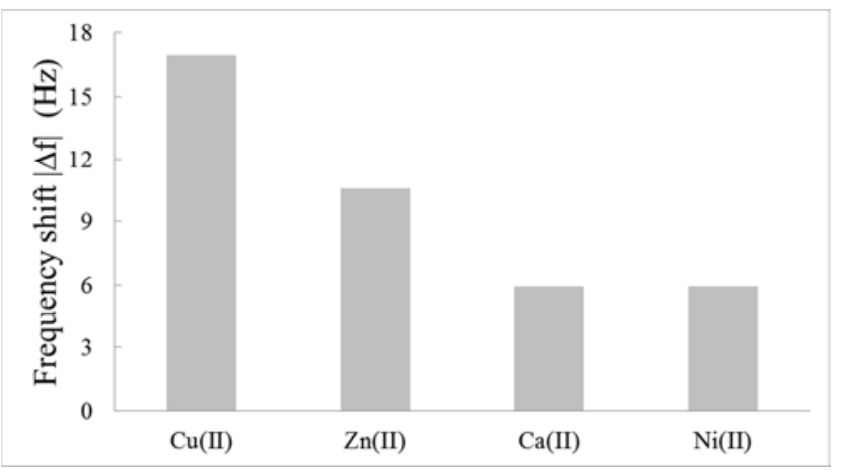

Figure 9. Selectivity of the QCM sensor with a B/A volume ratio of 9 to various metal ions at $10 \mu \mathrm{M}$.

\subsection{Characteristics of Metal Ion Detection}

The sensing performance of the developed QCM sensor obtained by monitoring the frequency shift that was caused by the presence of $\mathrm{Cu}$ (II) ions. Each injection of $\mathrm{Cu}$ (II) solution, flowed over the surface of the developed QCM sensor, reduced the resonant frequency because the $\mathrm{Cu}$ (II) ions interacted with the sensing film binding sites. Then, the $\mathrm{Cu}$ (II) ions were washed away from the sensing film rapidly by dionized water, partially returning the resonant frequency 
to its initial value. Therefore, this developed QCM sensor based on mixture of phosphate-modified dendrimer and ionophore exhibited partially reversible detection of the $\mathrm{Cu}$ (II) ion. Figure 10 plots the change in resonant frequency of the developed QCM sensor with a B/A volume ratio of 9 as a function of the concentration of $\mathrm{Cu}(\mathrm{II})$ ions. The sensitivity of the sensor to the $\mathrm{Cu}$ (II) ions concentration is roughly linear with $\mathrm{R}^{2}=0.9025$ in a logarithmic coordinate system.

Table 2 lists the results of previous studies for detecting $\mathrm{Cu}(\mathrm{II})$ ions in solution $[30,31]$. These QCM sensors developed in this study had also short response time that was about 40 seconds. This QCM sensor developed in this study provides a good candidate for the detection of $\mathrm{Cu}$ (II) ions.

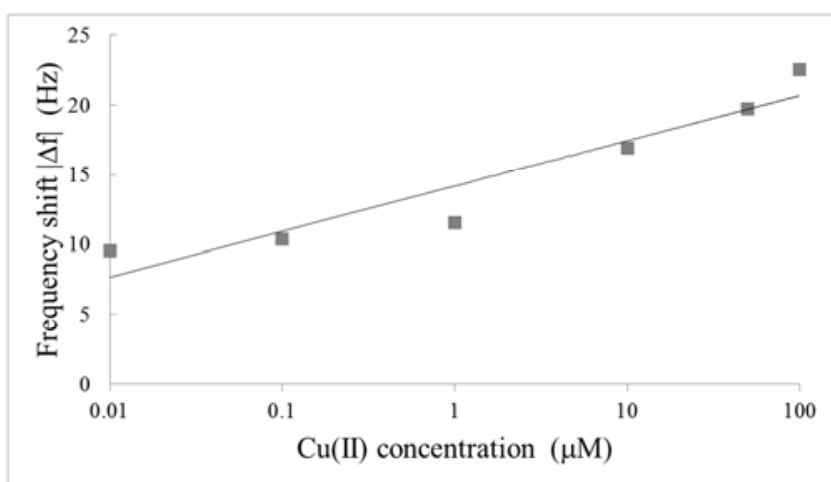

Figure 10. Dependence of change in resonant frequency of the QCM sensor, prepared with a $B / A$ volume ratio of 9 on concentration of $C u(I I)$ ions.

Table 2. Previous research for detecting $\mathrm{Cu}(\mathrm{II})$ ions in solution.

\begin{tabular}{|c|c|c|c|c|}
\hline Sensor & Sensitivity & Linear range & $\begin{array}{l}\text { Response } \\
\text { time }\end{array}$ & Reference \\
\hline MIP-based QCM & $1.5 \mathrm{~Hz} / \mu \mathrm{M}$ & $0.001-50 \mu \mathrm{M}$ & $6 \mathrm{~min}$ & [30] \\
\hline 4-CP-SPEs & $11 \mathrm{nA} / \mathrm{M}$ & $0.0075-0.18 \mu \mathrm{M}$ & - & {$[31]$} \\
\hline This study & $1.4 \mathrm{~Hz} /$ decade & $0.01-100 \mu \mathrm{M}$ & $40 \mathrm{~s}$ & \\
\hline
\end{tabular}

\section{Conclusion}

In this study, a new sensing membrane of mixture of phosphate-modified dendrimer and ionophore has been synthesized. It has been applied for $\mathrm{Cu}$ (II) metal ion sensing by QCM sensor at room temperature. The linear sensing range of $\mathrm{Cu}$ (II) ion was from $0.01 \mu \mathrm{M}$ to $100 \mu \mathrm{M}$. This developed QCM showed short response time that was about 40 seconds. The experimental results indicated that this developed QCM sensor based on combining phosphate-modified dendrimer and ionophore could be used for direct detection of $\mathrm{Cu}(\mathrm{II})$ metal ion with high sensitivity, good selectivity, satisfactorily good linearity, and fast response. Therefore, the QCM sensor developed in this study provided specificity with suitable for real-time detection of $\mathrm{Cu}$ (II) ions in environmental samples.

\section{Acknowledgements}

The authors thank the Ministry of Science and Technology, Taiwan, for partially supporting this research under Contract
No. NSC 102-2221-E-214-003-MY3 and MOST 105-2221-E-214-003.

\section{References}

[1] G. Z. Sauerbrey, "Use of quartz crystal vibrator for weighting thin films on a microbalance," Z. Phys., vol. 155, 1959, pp. 206-22.

[2] K. K. Kanazawa and J. G. Gordon, "Frequency of a quartz microbalance in contact with liquid," Anal. Chem., vol. 57, 1985, pp. 1770-1771.

[3] K. N. Huang, C. Y. Shen, S. H. Wang, and C. H. Hung, "Development of QCM-based immunosensor for detecting alpha-fetoprotein," Instrumentation Science \& Technology, vol. 44, 2013, pp. 311-324.

[4] Y. G. Lee and K. S. Chang, "Application of a flow type quartz crystal microbalance immunosensor for real time determination of cattle bovine ephemeral fever virus in liquid," Talanta, vol. 65, 2005, pp. 1335-1342.

[5] J. Park, S. Kurosawa, H. Aizawa, S. Wakida, S. Yamada, and K. Ishihara, "Comparison of stabilizing effect of stabilizers for immobilized antibodies on QCM immunosensors," Sensor Actuat. B, vol. 91, 2003, pp. 158-162.

[6] T. M. P. Hewa, G. A. Tannock, D. E. Mainwaring, S. Harrison, and J. V. Fecondo, "The detection of influenza A and B viruses in clinical specimens using a quartz crystal microbalance," J. Virol. Methods, vol. 162, 2009, pp.14-21.

[7] H. Zeng, H. Wang, F. P. Chen, H. Y. Xin, G. P. Wang, L. Xiao, K. Song, D.S. Wu, Q. He, and G. L. Shen, "Development of quartz-crystal-microbalance-based immunosensor array for clinical immunophenotyping of acute leukemias," Anal Chem., vol.351, 2006, pp. 69-76.

[8] W. C. Tsai and I. C. Lin, "Development of a piezoelectric immunosensor for the detection of alpha-fetoprotein," Sens. Actuators B, vol. 106, 2005, pp. 455-460.

[9] S. Kurosawa, J. W. Park, H. Aizawa, S. Wakida, H. Tao, and K. Ishihara, "Quartz crystal microbalance immunosensors for environmental monitoring," Biosens. Bioelectron, vol. 22, 2006, pp. 473-481.

[10] P. B. Tchounwou, C. G. Yedjou, A. K. Patlolla, and D. J. Sutton, "Heavy Metals Toxicity and the Environment," Molecular, Clinical and Environmental Toxicology, vol. 101, 2012, pp. 133-164.

[11] O. Zagurskaya-Sharaevskaya and I. Povar, "Determination of $\mathrm{Cu}$ (II) ions using sodium salt of 4-phenylsemicarbazone 1,2naphthoquinone-4-sulfonic acid in natural and industrial environments," Ecological Processes, vol. 4, 2015, pp. 1-5.

[12] M. Qadir, M. S. Javier, and J. Blanca, "Environmental risks and cost-effective risk management in wastewater use systems," In: Wastewater, Springer, Netherlands, 2015, pp. 55-72.

[13] B. Hasan, A. Abbas, S.T. Mohammad, and K. Hosein, "Preparation and characterization of magnetic nanocomposite of Schiff base/silica/magnetite as a preconcentration phase for the trace determination of heavy metal ions in water, food and biological samples using atomic absorption," Talanta, vol. 97, 2012, pp. 87-95. 
[14] S. Pande, "Analytical applications of room-temperature ionic liquids: A review of recent efforts," Anal. Chim. Acta, vol. 556, 2006, pp. 38-45.

[15] A. K. Malik, V. Kaur, and N. Verma, "A review on solid phase microextraction-high performance liquid chromatography as a novel tool for the analysis of toxic metal ions," Talanta, vol. 68, 2006, pp. 842-849.

[16] J. S. Becker, M. Zoriy, A. Matusch, B. Wu, D. Salber, C. Palm, and J.S. Becker, "Bioimaging of metals by laser ablation inductively coupled plasma mass spectrometry," Mass Spectrom. Rev., vol. 29, 2010, pp. 156-175.

[17] D. Heitkemper, J. Creed, J. Caruso, and F. L. Fricke, "Speciation of arsenic in urine using high-performance liquid chromatography with inductively coupled plasma mass spectrometric detection," J. Anal. At. Spectrom., vol. 4, 1989, pp. 279-284.

[18] V. Chandrasekhar, S. Das, R. Yadav, S. Hossain, R. Parihar, G. Subramaniam, and P. Sen, "Novel chemosensor for the visual detection of copper (II) in aqueous solution at the ppm level," Inorg Chem., vol. 51, 2012, pp. 8664-8666.

[19] X. Guo, Y. Yun, V. N. Shanov, H. B. Halsall, and W. R. Heineman, "Determination of trace metals by anodic stripping voltammetry using a carbon nanotube tower electrode," Electroanal., vol. 23, 2011, pp. 1252-1259.

[20] R. S. Freire and L.T. Kubota, "Application of self-assembled monolayer-based electrode for voltammetric determination of copper," Electrochim. Acta, vol. 49, 2004, pp.3795-3800.

[21] E. Chow, E. L. S. Wong, T. Böcking, Q. T. Nguyen, D. B. Hibbert, and J. J. Gooding, "Analytical performance and characterization of MPA-Gly-Gly-His modified sensors," Sens. Actuators B, vol. 111-112, 2005, pp. 540-548.

[22] Z. Zhang, Z. Chen, C. Qu, and L. Chen, "Highly sensitive visual detection of copper ions based on the shape-dependent LSPR spectroscopy of gold nanorods," Langmuir, vol. 30, 2014, pp. 3625-3630.
[23] M. Ghaedi, F. Ahmadi, and A. Shokrollahi, "Simultaneous preconcentration and determination of copper, nickel, cobalt and lead ions content by flame atomic absorption spectrometry," Journal of Hazardous Materials, vol. 142, 2007, pp. 272-278.

[24] G. R. Newkome and C. D. Shreiner, "Poly(amidoamine), polypropylenimine, and related dendrimers and dendrons possessing different 1-2 branching motifs: an overview of the divergent procedures," Polymer, vol. 49, 2008, pp. 1-173.

[25] M. W. P. L. Baars and E. W. Meijer, "Host-guest chemistry of dendritic molcules, Topics in Current Chemistry", vol. 210, 2000, New York, USA, Springer, p. 318.

[26] I. Grabchev, D. Staneva, S. Dumas, and J. M. Chovelon, "Metal ions and protons sensing properties of new fluorescent 4-N-methylpiperazine-1, 8-naphthalimide terminated poly(propyleneamine) dendrimer," J. Mol. Struct., vol. 999 2011, pp. 16-21.

[27] S. H. Wang, C. Y. Shen, Y. M. Lin, and J. C. Du, "Piezoelectric sensor for sensitive determination of metal ions based on the phosphate-modified dendrimer," Smart Materials and Structures, vol. 25, 2016, pp. 085018 (8pp).

[28] L. Rodriguez-Pardo, J. Fariña, C. Gabrielli, H. Perrot, and R. Brendel "Resolution in quartz crystal oscillator circuits for high sensitivity microbalance sensors in damping media," Sens. Actuators B, vol. 103, 2004, pp. 318-324.

[29] X. M. Du, S. H. M Chiu, D. H. C Ong, R. Vellaisamy, and M. H. W Lam, "Metal ion-responsive photonic colloidal crystalline micro-beads with electrochemically tunable photonic diffraction colours," Sens. Actuators B, vol. 223, 2016, pp. 318-323.

[30] C. Y. Shen, Y. M. Lin, and R. C. Hwang, "Detection of Cu(II) ion in water using a quartz crystal microbalance," Journal of Electrical and Electronic Engineering, vol. 4, 2016, pp. 13-17.

[31] Z. P. Yang and C. J. Zhang, "Designing of MIP-based QCM sensor for the determination of $\mathrm{Cu}(\mathrm{II})$ ions in solution," Sens. Actuators B, vol. 142, 2009, pp. 142-210. 